\title{
SCIENTIFIC REPORTS

\section{On-site single pollen metabolomics reveals varietal differences in phosphatidylinositol synthesis under heat stress conditions in rice}

\author{
Hiroshi Wada $\mathbb{( 1}^{1,6^{*}}$, Yuto Hatakeyama $\mathbb{1}^{1,6}$, Taiken Nakashima $\mathbb{( i )}^{2}$, Hiroshi Nonami $\mathbb{1}^{3}$, \\ Rosa Erra-Balsells $\mathbb{D}^{4}$, Makoto Hakata $\mathbb{1}^{1}$, Keisuke Nakata $\mathbb{D}^{3}$, Kenzo Hiraoka $\mathbb{D}^{5}$, \\ Yayoi Onda $\mathbb{1}^{3}$ \& Hiroshi Nakano $\mathbb{1}^{1}$
}

Although a loss of healthy pollen grains induced by metabolic heat responses has been indicated to be a major cause of heat-induced spikelet sterility under global climate change, to date detailed information at pollen level has been lacking due to the technical limitations. In this study, we used picolitre pressure-probe-electrospray-ionization mass spectrometry (picoPPESI-MS) to directly determine the metabolites in heat-treated single mature pollen grains in two cultivars, heat-tolerant cultivar, N22 and heat-sensitive cultivar, Koshihikari. Heat-induced spikelet fertility in N22 and Koshihikari was $90.0 \%$ and $46.8 \%$, respectively. While no treatment difference in in vitro pollen viability was observed in each cultivar, contrasting varietal differences in phosphatidylinositol (PI)(34:3) have been detected in mature pollen, together with other 106 metabolites. Greater PI content was detected in N22 pollen regardless of the treatment, but not for Koshihikari pollen. In contrast, there was little detection for phosphoinositide in the single mature pollen grains in both cultivars. Our findings indicate that picoPPESI-MS analysis can efficiently identify the metabolites in intact single pollen. Since $\mathrm{PI}$ is a precursor of phosphoinositide that induces multiple signaling for pollen germination and tube growth, the active synthesis of $\mathrm{PI}(34: 3)$ prior to germination may be closely associated with sustaining spikelet fertility even at high temperatures.

In rice, extremely high temperature conditions at flowering have caused widespread yield instability across many production areas, due to heat-induced spikelet sterility under global warming ${ }^{1-3}$. It has been accepted that exposure to temperatures higher than $35^{\circ} \mathrm{C}$ at flowering induces spikelet sterility, leading to yield loss based on growth chamber experiments ${ }^{4}$. Many researchers have put into efforts to study the underlying mechanisms behind spikelet sterility. It has been reported that the ability of pistil to be fertilized remained unaffected when exposed to high temperatures below $41^{\circ} \mathrm{C}$, and a loss of heat-induced fertility was mostly attributed to decreased pollen viability and disturbed pollen shedding, resulting in the reduction in germinated pollen grains on a stigma ${ }^{4}$. A decline in population of healthy pollen grains has gained attention as a cause of spikelet sterility ${ }^{5}$, as well as anther indehiscence ${ }^{4,6}$. Although multiple quantitative trait loci (QTL) have been identified for heat tolerance at flowering ${ }^{7}$, information is still limited and no superior heat-tolerant cultivars at flowering have been developed, at least in $\operatorname{Japan}^{8}$. For reasons of convenience and rapidness, in vitro pollen viability has been widely determined by using iodine staining for screening in rice breeding; however, this method has been recently called into question ${ }^{1}$. As the heat risk is expected to intensify with respect to global climate change, it is highly desirable to speed up rice breeding through identification of more reliable molecular marker(s). However, it seems likely that much less attempts have been conducted in the view of pollen physiology in rice.

${ }^{1}$ Kyushu Okinawa Agricultural Research Center, National Agriculture and Food Research Organization, 496 Izumi, Chikugo, Fukuoka, 833-0041, Japan. ' Faculty of Agriculture, Hokkaido University, Kita-9 Nishi-9, Kita-Ku, Sapporo, 060-8589, Japan. ${ }^{3}$ Graduate School of Agriculture, Ehime University, 3-5-7 Tarumi, Matsuyama, 790-8566, Japan. ${ }^{4}$ Department of Organic Chemistry, University of Buenos Aires, Buenos Aires, 1428, Argentina. ${ }^{5}$ Clean Energy Research Center, The University of Yamanashi, 4-3-11 Takeda, Kofu, Yamanashi, 400-8511, Japan. ${ }^{6}$ These authors contributed equally: Hiroshi Wada and Yuto Hatakeyama. *email: hwada@affrc.go.jp 


\begin{tabular}{|l|r|l|l|}
\hline & Cultivar & Treatment & Cultivar $\times$ treatment \\
\hline Spikelet fertility & $<0.001$ & $<0.001$ & $<0.001$ \\
\hline Anther length & 0.726 & 0.168 & 0.882 \\
\hline Pollen grain diameter & $<0.001$ & 0.885 & 0.546 \\
\hline In vitro pollen viability & 0.821 & 0.061 & 0.485 \\
\hline
\end{tabular}

Table 1. Two-way analysis of variance (ANOVA) of the effects of cultivar, heat treatment and their interaction on spikelet fertility, anther length, pollen grain diameter, and in vitro pollen viability in growth chamber-grown rice. The F-value probabilities at $95 \%$ confidence are indicated.

Pollen grains are male microgametophytes, consisting of the vegetative and the generative cells. The generative cell divides to form twin sperm cells, and the resulting male gametophyte is called the tricellular pollen'. Upon pollen germination, the pollen tube extends and grows down toward the ovule, and two sperm nuclei enter the female gametophyte and participate in a double fertilization process. According to the early microscopic observation $^{10}$, the developmental stage of rice pollen before flowering corresponds to the mature tricellular pollen, where starch degradation progressively occurs prior to anther dehiscence. Once the anther has dehisced, pollen falls on to the stigma, followed by pollen hydration and germination on the stigma ${ }^{11}$. Rice-like anemophilous pollen accumulate mostly starch, lipid, and proteins required for germination and pollen tube growth. During the last period of development, pollen accumulates two types of lipids, the neutral lipids stored in oil lipids and the polar lipids in the form of densely packed membranes ${ }^{12}$. The neutral lipids, including triacylglycerol, derived from the diploid tapetal cells, were closely associated with both desiccation tolerance and pollen adhesion to the stigma. In contrast, the polar lipids including membrane-associated phospholipids formed by the haploid vegetative cells would be used for pollen tube growth ${ }^{12,13}$.

There is accumulating evidence that inositol-containing lipids, phosphoinositide species can promote pollen hydration, germination and tip growth through multiple signaling ${ }^{12,14-16}$. One of the phospholipids, called phosphatidylinositol (PI), is known to be generated by catalyzing the transfer of free inositol to the backbone of a glycerophospholipid through the activation of PI synthase (EC 2.7.8.11) in the endoplasmic reticulum as the phosphoinositide precursor. In sorghum, it has been reported that changes in pollen phospholipids accompanied by an increase in reactive oxygen species (ROS) at high night temperatures may be closely associated with the reduction in pollen function ${ }^{17}$. By using lipidome analysis in pooled wheat pollen, it has been shown that C34:3 and C36:6 species are predominant phospholipids ${ }^{17}$, although the exact chemical composition of the predominant phospholipids has not been identified, implying the requirement of high-resolution cell metabolomics. So far, little is known about the possible changes in phospholipid biosynthesis in rice pollen in response to heat. However, if rice pollen exhibits similar heat responses, then PI synthesis may be the prerequisite for turning on the signaling even under heat conditions. In this work, we have hypothesized that PI biosynthesis in the mature pollen is closely associated with rice heat tolerance for spikelet sterility. Given that lipid metabolism is highly sensitive to temperature change, determining cytosolic metabolite(s) in the single pollen grain being exposed to heat would be ideal but challenging. Furthermore, methods to do the required analysis under heat-like temperature stress were not available until recently.

We have used a newly developed on-site cell-specific analysis (see Fig. S1 in Wada et al. 2019) ${ }^{18}$ to make this analysis possible. This analytical method consists of single cell metabolomics, called 'picolitre pressure-probe-electrospray-ionization mass spectrometry (picoPPESI-MS)'19 and accurate environmental control. The picoPPESI-MS technique has been successfully applied to several cell-specific research in different plant tissues, such as tomato trichomes ${ }^{19}$, rice endosperms ${ }^{18}$, and developing xylems in Norway spruce ${ }^{20}$. In this study, we have directly inserted the tip of a finely tapered quartz capillary tip into the developing pollen grains in intact plants growing under controlled high temperature environments to identify the internal metabolites. Two varieties, heat-tolerant cultivar, $\mathrm{N} 22^{21}$ and heat-sensitive cultivar, Koshihikari ${ }^{22}$, have been provided to test our hypothesis. Here, we show that there was a clear varietal difference in PI content in developing ungerminated pollen, which may be closely associated with heat tolerance and contribute to the steady spikelet fertility. Several heat-induced metabolic changes in PI-related pathway will be discussed.

\section{Results}

Spikelet fertility under heat conditions. Two-way ANOVA revealed significant cultivar and heat temperature effects as well as a significant effect of their interaction (Table 1). Spikelet fertility in the Koshihikari control group was ca. 96\%, although there was a remarkable decline in fertility of Koshihikari spikelets when exposed to heat conditions, reaching $46.8 \%$ (Fig. 1A). In contrast, spikelet fertility in N22 control declined slightly under heat conditions, but with no significant treatment difference (Fig. 1A).

Anther length, pollen grain morphology and in vitro viability under heat conditions. There were no varietal and treatment effects and no significant interaction in anther length (Fig. 1B, Table 1). According to two-way ANOVA, a significant cultivar effect was observed in the pollen grain diameter, but not for both treatment effect and the interaction (Table 1). The diameter in Koshihikari pollen grains was $44 \mu \mathrm{m}$ on average, whereas the mean diameter of N22 pollen grains was $35 \mu \mathrm{m}$, slightly smaller than that of Koshihikari (Fig. 1C). The number of pollen grains per anther in control in Koshihikari and N22 was 1002 and 1248 on average $(n=3)$, respectively, and a significant varietal difference was observed by $t$-test $(P=0.02)$. Although in vitro pollen viability was prone to decline under heat conditions regardless of cultivars, but with no significant cultivar and treatment effects and the significant interaction between the two factors (Fig. 1D, Table 1). 


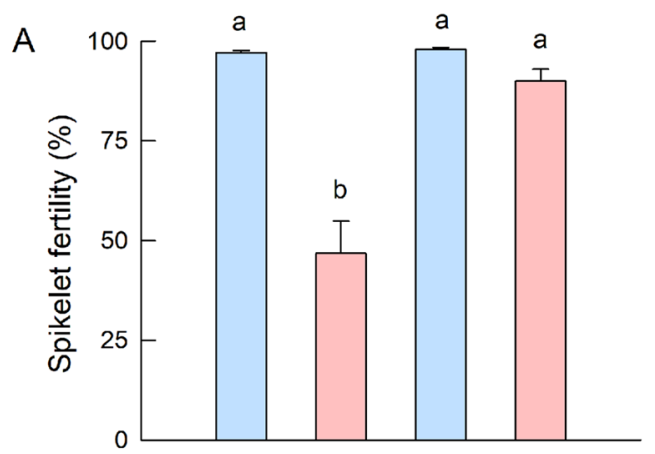

$\mathrm{B}$
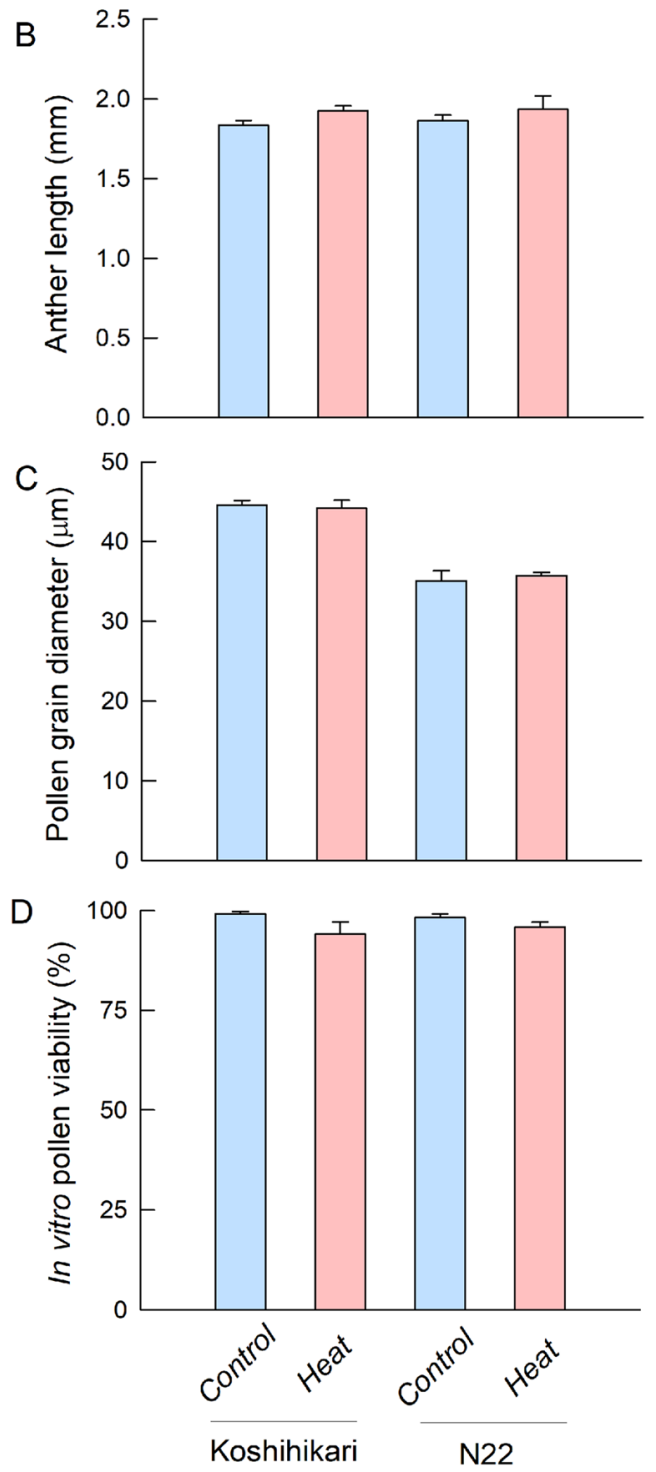

Figure 1. Spikelet fertility (A), anther longitudinal length (B), pollen grain diameter $(\mathbf{C})$, and in vitro pollen viability (D) in Koshihikari and N22 grown under high temperature conditions for $48 \mathrm{~h}$ after heading in the growth chambers. Data in $(\mathbf{A}, \mathbf{B}, \mathbf{D})$ are means $( \pm \mathrm{SE})$ of 3-4 replicates. Data in C are means $( \pm \mathrm{SE})$ of 3-6 replicates. Means followed by different letters indicate significant differences between treatments according to two-way ANOVA followed by Tukey's test $(P \leq 0.05)$.

Profiling pollen metabolites under normal conditions. By using the analytical method, 107 metabolites in total (133 signals in total including cluster ions), including organic acids, amino acids, carbohydrates, cell wall-related materials, and hormones, such as gibberellin A3 $(\mathrm{m} / z 345)$, trans-zeatin $(\mathrm{m} / z 218)$, and salicylic acid $(\mathrm{m} / z$ 137), have been simultaneously detected in the single pollen grains (Fig. 2A,C, Table S1). In both cultivars, 

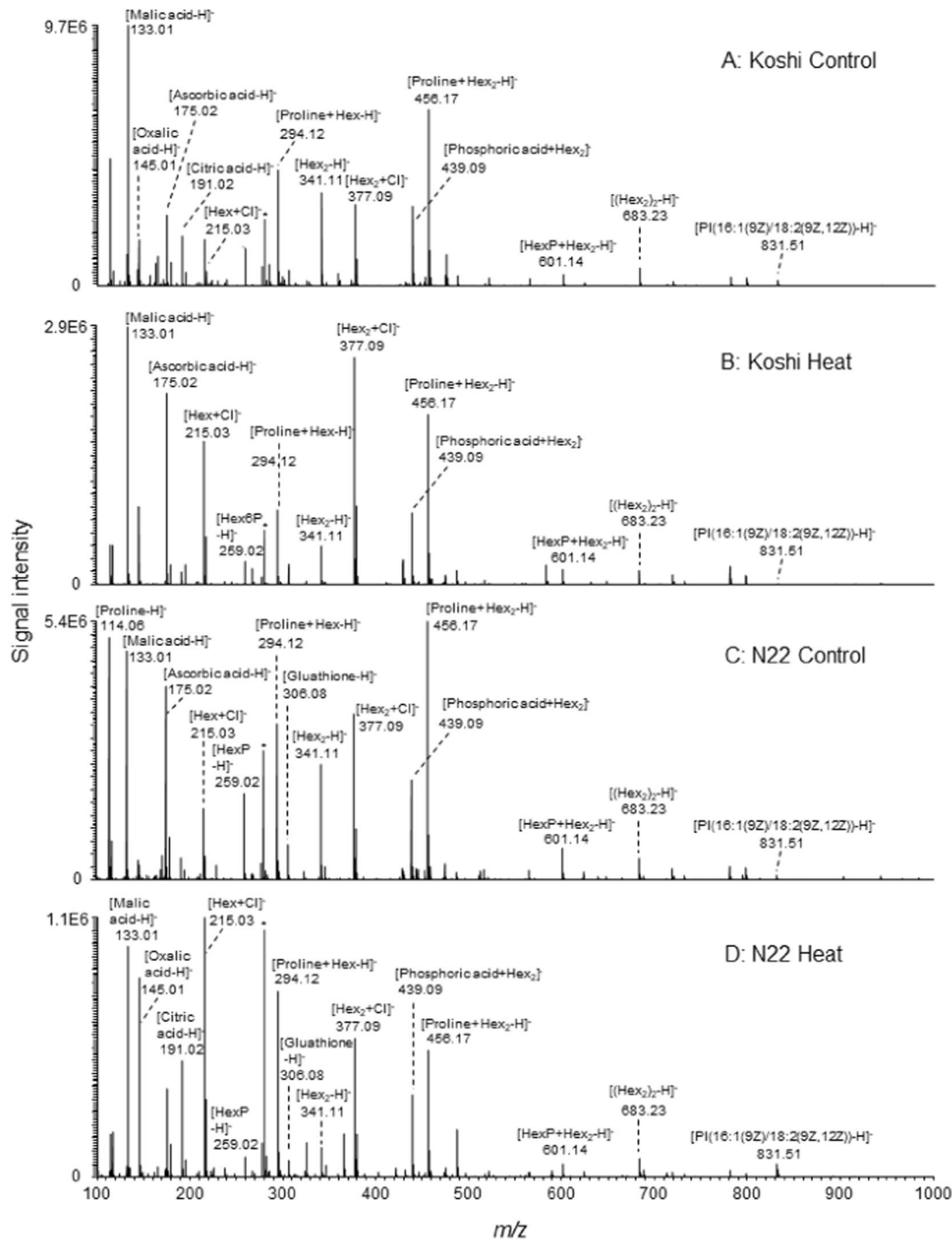

Figure 2. PicoPPESI mass spectra in negative ion mode obtained from the single pollen grains for the different treatments. The data are representative of repeated experiments with 8-16 pollen grains in total from 4-5 plants in each treatment. Asterisk indicates a cluster from ionic solution used.

the peaks of proline $(\mathrm{m} / \mathrm{z} 114)$, ascorbic, citric, malic, and 2-oxoglutaric acids (as $[\mathrm{M}-\mathrm{H}]^{-}, \mathrm{M}=$ molecular species), sugars (as $[\mathrm{M}-\mathrm{H}]^{-}$and/or $[\mathrm{M}+\mathrm{Cl}]^{-}$), and sugar-organic acid clusters (as $\left[\mathrm{M}^{\prime}-\mathrm{H}\right]^{-}, \mathrm{M}^{\prime}=$ summation of individual molecular mass of chemical cluster component species) were identified as major ions (Fig. 2A-D, Table $\mathrm{S} 1)$. N22 pollen grains were prone to accumulate greater amount of succinic acid $(\mathrm{m} / z 117)$, pentose $(\mathrm{m} / z$ 149, significant at $P>0.08)$, Hex $(m / z 179,215)$, proline $(m / z 114)$, and their cluster ions ([Proline $+\mathrm{Hex}-\mathrm{H}]^{-}(\mathrm{m} / z$ 294), [Proline $\left.\left.+\mathrm{Hex}_{2}-\mathrm{H}\right]^{-}(\mathrm{m} / z 456)\right)$, glutathione $(m / z 306), \mathrm{Hex}_{2}\left(m / z 377\right.$ as $\left.[\mathrm{M}+\mathrm{Cl}]^{-}\right), \mathrm{AMP}(\mathrm{m} / z 346)$, glycerol 3-phosphate $(\mathrm{m} / z$ 171), $\mathrm{PI}(\mathrm{C} 34: 3)(\mathrm{m} / z$ 832), phosphatidylethanolamine, $\mathrm{PE}(\mathrm{C} 34: 3)(\mathrm{m} / z 712)$, phosphatidic acid, $\mathrm{PA}(\mathrm{C} 34: 3)(\mathrm{m} / z$ 669), and most fatty acids, compared with Koshihikari (Fig. 2, Table S1). In addition, HexP $\left(\mathrm{m} / z\right.$ 259) and $\mathrm{HexP}_{2}(\mathrm{~m} / z$ 338) were frequently detected, but there was no or little detection for other hexose phosphates, such as $\operatorname{HexP}_{3}(m / z 418)$ and $\operatorname{HexP}_{4}(m / z$ 499)(Table S1). The signal for cysteine (Cys) $(m / z 120$ as $[\mathrm{Cys}-\mathrm{H}]^{-}$) was also observed in control of both cultivars (Table S1). 


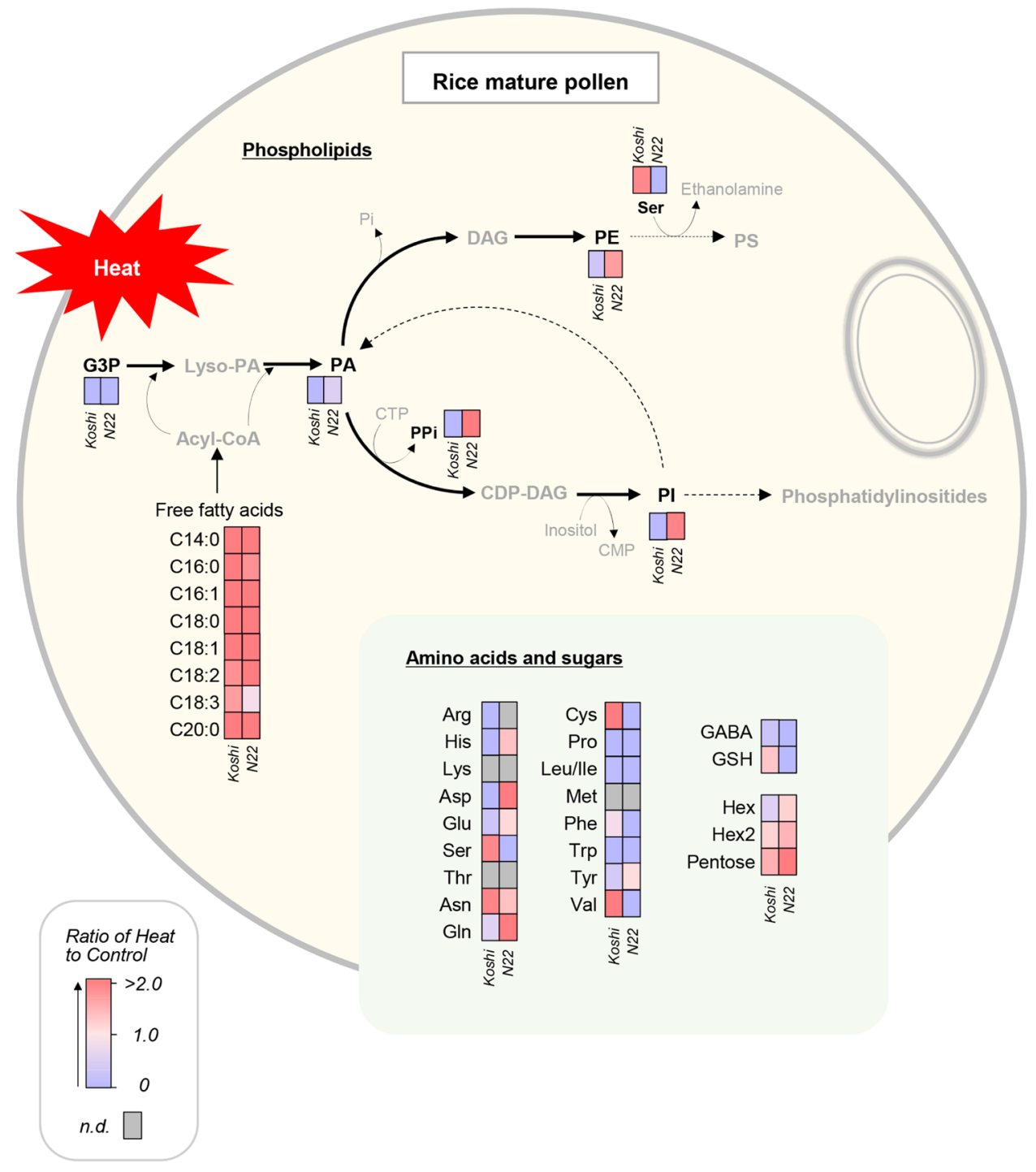

Figure 3. Putative phosphatidylinositol (C34:3) biosynthesis pathway and free fatty acids, amino acids, and sugars detected in the rice single mature pollen under heat conditions. The ratios of heat treatment to control in each cultivar are shown with a color scale. Trace amounts or absences of metabolites detected at less than $60 \%$ of frequency were shown in either gray letters and boxes. In each box set, Koshihikari and N22 are shown in left and right boxes, respectively.

Profiling pollen metabolites under heat conditions. When exposed to heat, the content of phospholipid synthesis-related metabolites, glycerol-3-phosphate and PA(34:3) declined in both cultivars (Table S1). However, the decline in PA(34:3) in N22 was much smaller than that of Koshihikari under heat conditions, and significant varietal difference was observed $(P=0.02)$. There were clear varietal differences in the accumulation pattern of the predominant $\mathrm{PI}(34: 3)$ and $\mathrm{PE}(34: 3)$ isomers in response to heat (Figs. 2 and 3, Table S1). Under the heat conditions, greater accumulation was observed in N22 for the predominant PI(34:3) and PE(34:3) isomers, but its content in Koshihikari was contrastingly declined. And consequently, there were significant varietal differences in heat-treated pollen at $P=0.13$ and $P=0.21$, respectively for the content of $\mathrm{PI}(34: 3)$ and $\mathrm{PE}(34: 3)$ isomers. The detected PI(34:3) isomer was shown to be composed of palmitoleic acid, C16:0 and linolenic acid, C18:3 by using MS/MS analysis (Fig. S2). PA(C36:6), PI(C36:6), and PE(C36:6) signals were less frequently detected in N22, compared with C34:3 species, but not for PC(36:6)(Table S1). In both cultivars, most fatty acids increased by heat, except for linolenic acid $(\mathrm{m} / z$ 277) in N22 (Fig. 2, Table S1). The ratio of C18:2 to C18:3 between control and heat treatment in Koshihikari changed little (4.25 and 3.94, respectively), although that in N22 dramatically declined from 13.67 down to 1.61 .

Changes in these phospholipid signals including PI signals (C34:3 and C36:6) and their varietal differences were not similarly observed in the anther tissue samples (Fig. S1). There was little to no detection of phosphoinositide species, such as phosphatidylinositol-phosphate and phosphatidylinositol-bisphosphate, as well as other lipid-related metabolites including diacylglycerol (DAG), cytidine diphosphate (CDP), CDP-ethanolamine, 
PG(34:3), PIP3(34:3), PI(3)P(34:3), choline, CDP-choline (Citicoline), PIP2(34:3), and PIP2(36:6) in each treatment at the corresponding stage of development (see Table $\mathrm{S} 1)$.

The content of Cys, serine (Ser) $(\mathrm{m} / z$ 104), and threonine $(\mathrm{m} / z$ 118) increased in heat-treated Koshihikari pollen grains, whereas serine $(\mathrm{m} / z 104)$ and threonine $(\mathrm{m} / z$ 118) signals declined substantially in heat-treated N22 pollen grains, but with little detection of Cys-related signals $(n=14$, Table S1). Similar patterns were observed between cultivars for Cys-sugar cluster ions, such as $[\mathrm{Cys}+\mathrm{Hex}-\mathrm{H}]^{-}$and $\left[\mathrm{Cys}+\mathrm{Hex}_{2}-\mathrm{H}\right]^{-}$(not detected and $7.1 \%$, respectively in N22 pollen). The content of dehydroascorbic acid $(\mathrm{m} / z 173)$, oxaloacetic acid $(\mathrm{m} / z 131)$, citric acid $(\mathrm{m} / z 191)$, aspartic acid $(\mathrm{m} / z 132)$, glutamine $(\mathrm{m} / z 145)$, and methyl jasmonate $(\mathrm{m} / z 223)$ in Koshihikari decreased at high temperatures, whereas an increase in content of these metabolites and a cytokinin, presumably trans-zeatin $(\mathrm{m} / z 218)$ was observed in N22 (Table S1). For cell wall-related metabolites, the content of UDP $(\mathrm{m} / z$ $402)$, coumaryl-alcohol $(\mathrm{m} / z \mathrm{149})$, and $\alpha$-L-rhamnose $(\mathrm{m} / z 163)$ decreased in heat-treated Koshihikari, although those in N22 increased (Table S1).

\section{Discussion}

In this work, we hypothesized that PI biosynthesis may be closely associated with heat tolerance in rice pollen for spikelet fertility. We collected picolitre pollen fluids from a growing single rice pollen grain to conduct on-site real-time metabolomics using picoPPESI-MS (Fig. 4). We have characterized changes in pollen metabolites in response to heat stress, as demonstrated by two cultivars exhibiting different spikelet fertility under heat conditions (Fig. 1A). At high temperatures, the content of PA declined in both cultivars and no phosphoinositide accumulation was observed at the mature stage of development; however, very clear and contrasting varietal differences in the accumulation of PI, composed of C16:0 and C18:3 (see Fig. S2), have been revealed prior to anther dehiscence and pollen germination (Figs. 2 and 3). Regardless of the application of heat treatment, heat tolerant cultivar, N22 exhibited greater PI accumulation than Koshihikari (Fig. 3), suggesting a close relation to maintaining high spikelet fertility (Fig. 4). Given into the fact that PI is a precursor of phosphoinositide species that induces multiple signaling as regulators of pollen hydration, germination, and tube growth ${ }^{12,14-16}$, we conclude that an active PI biosynthesis, detected at the single pollen grain level using picoPPESI-MS, is essential for male gametophyte development prior to the initiation of multiple signaling and contributes to heat tolerance for sustaining spikelet fertility under high temperature conditions.

Using a cell pressure probe, the direct turgor measurement has been made in a model plant, Lilium longiflorum pollen grains having typically $100 \mu \mathrm{m}$ diameter ${ }^{23}$ and pollen tubes exhibiting between $100-1600 \mu \mathrm{m}$ of tube length $^{24}$. It seems that there have been no other pollen-related pressure probe works conducted in other species. In this work, we have directly inserted the microcapillary tip into growing rice pollen grains having 31-53 $\mu$ m diameter (i.e., 15.3-77.4 pL)(see Fig. 4A), corresponding to $2.9-14.7 \%$ of Lilium pollen grain volume. Benkert et al. showed good pressure tracing over $15 \mathrm{~min}$ in lily pollen ${ }^{24}$. In the case of intact rice mature pollen examined here, it was possible to observe the rapid backward movement of oil/cell sap boundary (meniscus) in the microcapillary immediately after tip impalement (see Fig. 4A), indicating that pollen grains could exhibit positive turgor (i.e., internal hydraulic pressure) in mature pollen. However, it was not attainable to detect turgor accurately with a good stability or maintaining the meniscus location for long duration ${ }^{25}$, because of the relatively small pollen volume and occurrence of tip plugging during the meniscus oscillation due to the high viscosity of the pollen fluids in all treatments examined.

Recently, it has been pointed that most analyses conducted in the anthers or even flowers have been often regarded as the phenomenon confined to the pollen ${ }^{26}$. Despite of the impact on agricultural science, no attempts have been made for conducting metabolomics on pollen grains attached to growing crop plants. In this study, single pollen metabolomics using picoPPESI-MS has revealed the key pathway associated with heat tolerance (Figs. 2 and 3). Because of the high dilution rate (35-folds), it is postulated that the diluted fluids would be homogeneous in the capillary prior to electrospray ionization (Fig. 4C). The mass analyzer could quantify the amount of each metabolite ion in relation to the amount of the most abundant ion to give the relative abundance. Therefore, possible variations from the difference of dilution rate are likely to be ignorable as far as data analysis is on the relative abundance basis. Given into that considerable spatial heterogeneity in organelle arrangement occurs in mature rice pollen at polarity formation ${ }^{10,27}$, it is not surprising that slight differences in the insertion location of the target pollen grain might have caused some variations in MS spectra (see $p$-values in Table S1).

As well as other species, a series of physiological changes dramatically occurs in mature rice pollen prior to anther dehiscence and germination. As a result of the rapid decline in water content ( $86 \%$ down to $75 \%$ ) accompanied by starch degradation, the concentration of sugars, amino acids, and inorganic phosphorous progressively increased under optimal temperature conditions ${ }^{28}$, although data on heat-induced pollen metabolisms, particularly internal lipids is lacking. In pooled wheat pollen, Narayanan et al. conducted lipidome analysis in heat-treated cultivars to report that $\mathrm{C} 34: 3$ and $\mathrm{C} 36: 6$ species dominated the composition of extraplastidic phospholipids including PI, although neither varietal differences in composition nor alteration of pollen lipidome was observed $^{29}$. In this study, we have conducted single pollen metabolomics to increase the resolution, compared with conventional analysis. This allows us to find that there were clear varietal differences in heat responses on phospholipids, particularly for heat-induced accumulation of the predominant $\mathrm{PI}(34: 3)$ and $\mathrm{PE}(34: 3)$ in the heat-tolerant cultivar, N22 (Figs. 2 and 3, Table S1) that marked high spikelet fertility (Fig. 1A). The MS/MS data clearly showed that PI(34:3) is composed of C16:0 and C18:3 (Fig. S2). The secondary phospholipid C36:6 species was also detected (Table S1), consistent with ref. ${ }^{29}$. It is expected that the C36:6 species in our pollen samples may have had two C18:3 chains. Different from other fatty acids increased under heat conditions (Fig. 3), a remarkable reduction for the heat-to-control ratio of C18:3 signal confined in N22 is very likely to refer to the active synthesis of the both PI(34:3) and PI(36:6) and other related phospholipids, as detected simultaneously (Table S1). Importantly, these PI-related signals were not observed in the anther tissue samples (Fig. S1), suggesting that spatial localization was confined to pollen grains. Aside from PI synthesis, C16 and C18 fatty acids identified 

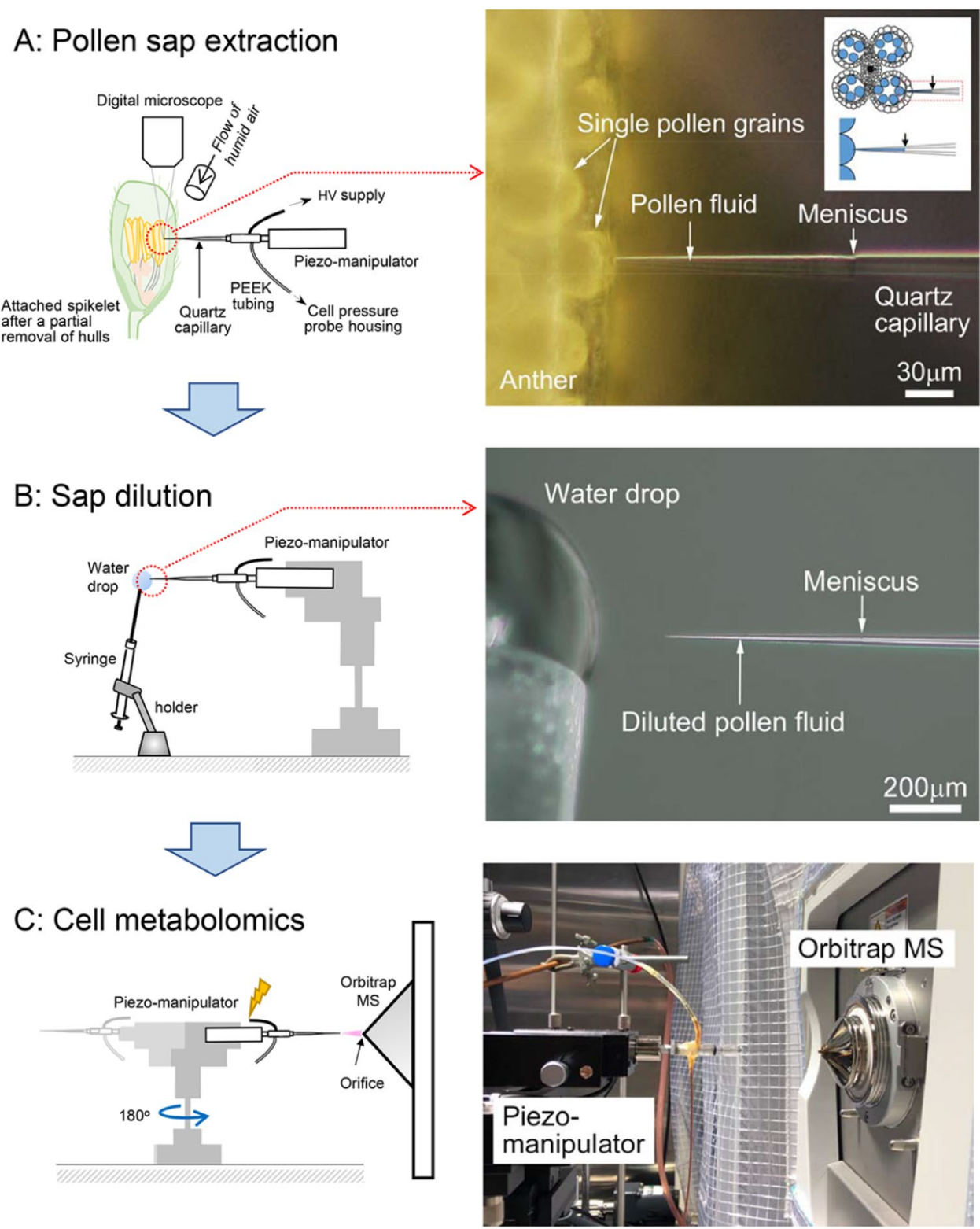

Figure 4. Illustrated workflow of on-site cell metabolomics in cellular fluids extracted from a single pollen grain located under the anther wall in intact plants which are exposed to heat conditions. The upper and lower insets in (A) shows a simple schematic of tip insertion into pollen on the anther cross section and anther side view, respectively. (A) shows a schematic of the fluid extraction and the microscopic image taken at the extraction. As soon as the cellular fluid was expelled into the capillary and the boundary (meniscus) was formed between the oil and cell sap, the tip was put into the water drop of ultrapure water for dilution (B). And then, the probe tip was immediately removed from the water drop and rotated $180^{\circ}$ using a $3 \mathrm{D}$ move/rotation micro-manipulator. And instantly, the metabolites in the fluids were analyzed in Orbitrap mass spectrometer by applying a high voltage without any pre-treatment (C, see Methods).

here may also be used for pollen exine formation in rice $^{30}$. Together with the above-mentioned lipids, it has been demonstrated that picoPPESI-MS is capable for detecting other metabolites, such as organic acids, amino acids, carbohydrates, cell wall-related materials, and hormones at the same time. These data indicate the robustness and usefulness of high-resolution on-site cell-specific analysis at single pollen level.

In general, mature pollen accumulate neutral lipids in lipid droplets, polar lipids in the form of densely packed membranes, and proteins required for tube growth ${ }^{12}$. Pollen lipid droplets are spherical organelles having a 0.5 to $2 \mu \mathrm{m}$ diameter, which is smaller than the tip size (see Methods). And therefore, it is assumed that numerous lipid droplets and membranes in the vicinity of ER would have contaminated in the picolitre pollen fluid during the collection. Shintaku et al. have shown that single cell can be lysed by applying a bipolar voltage pulse $(3 \mathrm{kV}$, $100 \mathrm{~ms}$ interval for each pulse) $)^{31}$. Regarding our picoPPESI-MS analysis, what exactly occurs during electrospray ionization remains to be investigated further in the view of analytical chemistry. However, given the fact that 
PI-like membrane phospholipids have been detected as strong signals in our analytical method, it is reasonably speculated that both lipid bodies and membrane that would be contaminated in our pollen fluid samples might be degraded in some manner when applying a high voltage $(-4 \mathrm{kV})$ for ionization to occur. If this is the case, then the observed heat responses including the compositional changes in phospholipid metabolism would partially explain the spatial changes in organelle compartmentation that precedes to germination.

There is concrete evidence indicating that biosynthesis of phosphoinositide species activates to promote germination and polar tube growth through multiple signaling ${ }^{12,14,15}$. All phosphoinositide species could derive from PI by phosphorylation of the lipid head group and are generated by one or two phosphorylation steps with phosphates catalyzing the reverse reactions. Therefore, significant PI accumulation observed in heat-treated N22 pollen could facilitate the forthcoming multiple signaling for pollen tube growth to succeed in double fertilization, resulting in the superior spikelet fertility observed in Fig. 4A. The reduction in PA (34:3) content and greater heat-induced pyrophosphate accumulation in N22 may support an activation of PI synthesis (Fig. 3). It is also anticipated that either activation in PI synthase $\mathrm{e}^{32}$ and/or the reduction in PI-specific phospholipase C activity ${ }^{33,34}$ may be the key enzymes regulating phospholipid metabolism to cope with heat. Alternative explanation is that considerable differences in PI turnover rate might exist between cultivars (Fig. 3). In N22, the rate of PI turnover might have slowed down to hinder phosphoinositide signaling from turning on pollen germination prior to anther dehiscence. If Koshihikari pollen had rapid PI turnover rate under heat conditions, PI might be degraded by phospholipase $\mathrm{D}$ to PA transformed into any other glycerophospholipid ${ }^{35}$. If this holds true, it is possible to interpret that in heat susceptible cultivars' pollen phospholipase D may be activated by heat. Any mutant of the PI synthase-encoding genes has not been reported yet ${ }^{12}$; however, our data strongly suggest that active biosynthesis and accumulation of the major PI (C34:3) is essential in the ungerminated rice pollen and is closely associated with spikelet fertility under heat conditions. Based on our finding, it is suggested that quantity and composition of PI may be used as an alternative method for evaluating pollen viability.

There is no direct evidence that increases in ROS scavenging activity sustains pollen development under heat conditions ${ }^{26}$. Pollen is known to accumulate numerous mitochondria, twenty times more than in vegetative cells ${ }^{36}$, and therefore pollen mitochondria may be the main source organelle of ROS produced during pollen development. In the present study conducted in single pollen, we have observed contrasting responses between cultivars in terms of TCA cycle metabolites, OAA, succinic, fumaric, and citric acids in response to heat (Table S1), suggesting that varietal difference in systematic ROS regulation may exist in pollen mitochondria. In this study, ROS signals, such as $\mathrm{H}_{2} \mathrm{O}_{2}$ themselves were undetectable because they were below the detection limit of Orbitrap MS (i.e., $m / z<50$ ); however, accumulation pattern for several redox metabolites, ascorbic acid and glutathione, consistently observed in all treatments (see control in Table S1), implies that certain ROS concentrations may be necessary for pollen maturation. In high night temperature-treated sorghum plants, it has been reported that changes in membrane-associated phospholipids accompanied by increasing ROS may be responsible for the reduction in pollen functions ${ }^{17}$. Likewise, there may be a close relation between PI-related synthesis and ROS concentration in rice pollen, which remains unexplored. However, it is noteworthy that pollen size was sustained under heat conditions in both cultivars (Fig. 4), presumably by osmotic adjustment as discussed below. And, it should be emphasized that opposite responses to heat for sugars, redox metabolites and Cys-related signals were observed between two cultivars (see Results, Table S1).

In general, Cys helps in the stabilization of the protein structure through the formation of disulfide bonds in the cells. More recently, we have used the same analytical method to report that cytosolic Cys accumulation occurred in heat-treated rice endosperms that reduced protein synthesis rate prior to chalky formation ${ }^{18}$. Similarly in rice pollen, our data is suggestive that heat tolerant rice cultivar pollen may have an effective mechanism for facilitating biosynthesis of proteins, such as phospholipid pathway-related enzymes and heat-shock proteins ${ }^{26}$, leading to the optimal pollen development prior to germination under heat conditions. If carbohydrate starvation were ongoing in heat-treated Koshihikari pollen, cytosolic Cys would contribute to increase in osmotic pressure (i.e., osmotic adjustment) to maintain pollen volume (Fig. 1B), presumably through a partial protein synthesis inhibition. Hence, the observed varietal differences in Cys-related signals may reflect the differences in the synthesis rate of proteins needed for germination. In this view, it is presumed that N22 pollen would be more effectively adjusted osmotically, compared with Koshihikari.

In conclusion, we have shown that the synthesis of a phospholipid, PI, directly detected in the mature rice pollen under heat conditions may be important for multiple signaling leading to polar tube growth after adhesion to the stigma and contribute to heat tolerance, maintaining spikelet fertility. We propose that PI biosynthesis may serve as an alternative biomarker for evaluating pollen viability, instead of conventional in vitro pollen viability using iodine staining. Further analysis may shed additional light on enhancing rice heat tolerance through the identification of regulatory gene(s) to high temperature conditions. As cell heterogeneity has been increasingly recognized as a biologically important phenomenon observable in plants, conducting cell metabolomics including picoPPESI-MS analysis may open new possibilities to reveal many more cell-specific physiological events in greater depth than more conventional tissue-level analysis.

\section{Methods}

Plant material. A growth-chamber experiment was conducted at the Kyushu Okinawa Agricultural Research Center, Chikugo, Japan. Rice seeds from two cultivars, a heat-susceptible cultivar, Oryza sativa L. 'Koshihikari'22 and heat-tolerant cultivar, $\mathrm{N} 22^{21}$ were provided. Each plant was grown in a plastic pot, as described previously ${ }^{37}$. They were grown by removing the tillers periodically to restrict each plant to its main culm to minimize sample-to-sample vaiations ${ }^{22,38}$ and cultivated in a cycle of day/night air temperatures of $26^{\circ} \mathrm{C}(13 \mathrm{~h}, 5: 50-18: 50) / 22^{\circ} \mathrm{C}$ $(11 \mathrm{~h}, 18: 50-5: 50)$ at $60 \%$ Relative humidity $(\mathrm{RH})$ until heading to rule out the potential effects of the history of heat conditions prior to heading. Two treatments were applied at heading: no heat as control $\left(26^{\circ} \mathrm{C}\right)$ and high temperature $\left(34^{\circ} \mathrm{C}\right)$. For the high-temperature treatment, pots were transferred to another growth chamber set 
at $34 / 28^{\circ} \mathrm{C}$ and $70 / 80 \% \mathrm{RH}$ at 9:00 on the day of heading. The photosynthetically active radiation (PAR) and the photoperiod were the same as the first growth chamber $\left(750 \mu \mathrm{mol} \mathrm{m}^{-2} \mathrm{~s}^{-1}\right.$ and $13 / 11 \mathrm{~h} \mathrm{day} /$ night, respectively). After $48 \mathrm{~h}$ of high-temperature treatment, the pots were transferred back to the control chamber to grow until they reached the mature stage of plants (40 days after heading; DAH). In total there were 14 pots in control and 16 pots in the heat treatment were prepared for each cultivar.

On-site cell metabolomics. By using picoPPESI-MS ${ }^{19}$, on-site cell metabolomics was conducted on single pollen grains of intact plants growing under heat conditions in the growth chambers (K260B029-S01, Tsubuku Corporation Ltd., Kurume, Japan), as reported in the previous study (see Fig. S1 in ref. ${ }^{18}$ ). A potted plant at the $2^{\text {nd }}$ day of heat treatment, corresponding to maturation stage of pollen grains ${ }^{10}$, was placed at the center of a U-shaped vibration-free table in the measurement room (see Fig. S1 in ref. ${ }^{18}$ ). The one of the first to third primary rachis branches, counted from the top of the intact panicle (i.e., the upper position in the panicle), were fixed on the sample holder with magnets, and then the top half of the lemma in the attached superior spikelets was quickly removed under humid conditions, and the attached anthers were gently fixed on the sample holder with surgical tape. Using a Piezo manipulator (DC-3K, Märzhäuser Wetzlar, Germany), the tip of microcapillary filled with a $0.01 \%$ (v/v) ionic liquid/silicone oil mixture ${ }^{19}$ was inserted into the developing pollen grains adjacent to the inner wall of the middle theca (Fig. 4A) to collect cellular fluid $(4.7 \pm 3.0 \mathrm{pL}$ [mean $\pm \mathrm{SD}, n=21])$. Immediately after the probe tip was moved into a water drop $(r=\mathrm{ca} .0 .7 \mathrm{~mm})$, and the fluids were diluted to avoid tip plugging at ionization by instantly reducing the oil pressure to sub-zero pressure (Fig. 4B). The probe tip was rotated and oriented toward the opening of an Orbitrap mass spectrometer (Q-Exactive, ThermoFisher Scientific Inc., MA, USA), and the tip was subsequently charged at $-4 \mathrm{kV}$ using a high-voltage generator (AKTB-05k1PN/S, Touwa Keisoku Corp., Tokyo, Japan) (Fig. 4C). The MS analysis was then conducted. Spikelets attached to the sample holder were humidified throughout the process, and all analyses were confined to superior spikelets in the upper position of panicle and the entire process of picoPPESI-MS analysis was completed within a few minutes per shot. Although humid conditions induced anther dehiscence, no septum rupture occurred during the above analysis. All manipulations were conducted under a digital microscope (KH-8700, HIROX Co. Ltd., Tokyo, Japan). The mass spectra reported here are representative of the repeated measurements on 8-16 pollen grains from 4-5 independent plants in each treatment.

Identification of phosphatidylinositol and anther sample analysis. Exact monoisotopic $m / z$ values for all the peaks on the mass spectra acquired were extracted using the Qual Browser application in the Thermo Xcalibur software (ThermoFisher Scientific). Metabolites were identified from the theoretical masses of candidate metabolites in the METLIN online metabolomics database (http://metlin.scripps.edu/index.php) allowing differences of $<5 \mathrm{ppm}$. In addition to the single pollen analyses described above, MS/MS analysis for PI(34:3) was conducted on N22 pollen grains in intact plants, together with standard L- $\alpha$-PI sodium salt from Glycine $\max$ (Sigma-Aldrich). Collision-induced dissociation (CID) tandem MS analysis was performed using the same Orbitrap MS coupled with the picoPPESI system, which allowed us the MS/MS identification of target molecules in the same picolitre pollen fluids. The MS scan was performed in negative ion mode with the same instrument settings as described above, except that the resolution was 70000. Additionally, MS analysis was conducted on the crude anther extracts collected from three plants in each treatment for both cultivars at the same stage. The anthers from two superior spikelets attached to the same position in a panicle were collected using forceps, frozen at $-80^{\circ} \mathrm{C}$ for $>2 \mathrm{~h}$, and freeze-dried. The sample was then mixed with $50 \%(\mathrm{v} / \mathrm{v})$ water $/ \mathrm{methanol}$ and sonicated. After centrifugation for $10 \mathrm{~min}$ at $10000 \mathrm{~g}$ at $4{ }^{\circ} \mathrm{C}$, the supernatant (i.e. crude tissue extract) was used for tissue MS analyses. All the standard chemicals and organic solvents used in the experiments, except for the standard PI from Glycine max, were LC/MS grade purchased from Wako Pure Chemical Industries, Ltd. (Osaka, Japan). Ultrapure water of $18.2 \mathrm{M} \Omega \mathrm{cm}^{-1}$ was used throughout the experiment.

Microscopy. By disrupting the developing anther samples from the superior spikelets attached to the same upper position in a panicle used for picoPPESI analysis, ungerminated mature pollen was collected. Pollen was viewed and photographed after being stained in potassium iodide for $1 \mathrm{~min}$, and in vitro pollen viability was determined. The anther length and pollen grain diameter on the light microscopic images were determined using ImageJ software (US National Institutes of Health). The values reported for pollen grain diameter represent the means of 3-6 independent plants (37-212 pollen grains per plant). For pollen number per anther and anther length, three plants were used in each treatment.

Spikelet fertility. After harvest, the panicles were dried at $30^{\circ} \mathrm{C}$ for 3 days. Thereafter, the number of mature seeds and empty caryopses was counted. The values reported for spikelet fertility represent the means of the spikelets attached to the same position from 3-4 independent plants (i.e., panicles) in each treatment.

Statistical analysis. For spikelet fertility, anther length, pollen grain, and in vitro pollen viability, data were subjected to two-way ANOVA (cultivar $\times$ treatment) to assess the effects of cultivar and treatment and their interaction on each parameter in JMP (version 12.1.0; SAS Institute Inc., Cary, NC, USA). Significant differences between means were then determined using the Tukey's test at $95 \%$ confidence $(P \leq 0.05)$. Analysis of all metabolome data was performed using Student's $t$ test in JMP.

\section{Data availability}

The datasets generated during and/or analyzed during the current study are available from the corresponding author on reasonable request.

Received: 14 November 2019; Accepted: 21 January 2020;

Published online: 06 February 2020 


\section{References}

1. Jagadish, S. V. K., Murty, M. V. R. \& Quick, W. P. Rice responses to rising temperatures-challenges, perspectives and future directions. Plant Cell Environ. 38, 1686-1698, https://doi.org/10.1111/pce.12430 (2015).

2. Morita, S., Wada, H. \& Matsue, Y. Countermeasures for heat damage in rice grain quality under climate change. Plant Prod. Sci. 19, 1-11, https://doi.org/10.1080/1343943X.2015.1128114 (2016).

3. Ishimaru, T., Hirabayashi, H., Sasaki, K., Ye, C. \& Kobayashi, A. Breeding efforts to mitigate damage by heat stress to spikelet sterility and grain quality. Plant Prod. Sci. 19, 12-21, https://doi.org/10.1080/1343943X.2015.1128113 (2016).

4. Satake, T. \& Yoshida, S. High temperature-induced sterility in indica rices at flowering. Jpn. J. Crop Sci. 47, 6-17, https://doi. org/10.1626/jcs.47.6 (1978).

5. Matsui, T., Omasa, K. \& Horie, T. The difference in sterility due to high temperatures during the flowering period among japonicarice varieties. Plant Prod. Sci. 4, 90-93, https://doi.org/10.1626/pps.4.90 (2001).

6. Matsui, T., Omasa, K. \& Horie, T. Mechanism of anther dehiscence in rice (Oryza sativa L.). Ann. Bot. 84, 501-506, https://doi. org/10.1006/anbo.1999.0943 (1999).

7. Ye, C. R. et al. Mapping QTL for heat tolerance at flowering stage in rice using SNP markers. Plant Breed. 131, 33-41, https://doi. org/10.1111/j.1439-0523.2011.01924.x (2012).

8. Wada, H. In Adaptation to Climate Change in Agriculture, (eds. Iizumi, T., Hirata, R. \& Matsuda, R.), https://doi.org/10.1007/978981-13-9235-1_3 (Springer, Singapore, 2019).

9. McCormick, S. Male gametophyte development. Plant Cell 5, 1265-1275, https://doi.org/10.1105/tpc.5.10.1265 (1993).

10. Raghavan, V. Anther and pollen development in rice (Oryza-sativa). Am. J. Bot. 75, 183-196, https://doi.org/10.2307/2443885 (1988).

11. Edlund, A. F., Swanson, R. \& Preuss, D. Pollen and stigma structure and function: The role of diversity in pollination. The Plant Cell 16, S84-S97, https://doi.org/10.1105/tpc.015800 (2004).

12. Ischebeck, T. Lipids in pollen - They are different. BBA-Mol. Cell Biol. L. 1861, 1315-1328, https://doi.org/10.1016/j. bbalip.2016.03.023 (2016).

13. Piffanelli, P., Ross, J. H. E. \& Murphy, D. J. Biogenesis and function of the lipidic structures of pollen grains. Sex. Plant Reprod. 11, 65-80, https://doi.org/10.1007/s004970050122 (1998).

14. Xue, H. W., Chen, X. \& Me, Y. Function and regulation of phospholipid signalling in plants. Biochem. J 421, 145-156, https://doi. org/10.1042/Bj20090300 (2009).

15. Zonia, L. \& Munnik, T. Osmotically induced cell swelling versus cell shrinking elicits specific changes in phospholipid signals in tobacco pollen tubes. Plant Physiol. 134, 813-823, https://doi.org/10.1104/pp.103.029454 (2004).

16. Gao, X. Q. \& Zhang, X. S. Metabolism and roles of phosphatidylinositol 3-phosphate in pollen development and pollen tube growth in Arabidopsis. Plant Signal. Behav. 7, 165-169, https://doi.org/10.4161/psb.18743 (2012).

17. Prasad, P. V. V. \& Djanaguiraman, M. High night temperature decreases leaf photosynthesis and pollen function in grain sorghum. Funct. Plant Biol. 38, 993-1003, https://doi.org/10.1071/Fp11035 (2011).

18. Wada, H. et al. Multiple strategies for heat adaptation to prevent chalkiness in the rice endosperm. J. Exp. Bot 70, 1299-1311, https:// doi.org/10.1093/jxb/ery427 (2019).

19. Nakashima, T. et al. Single-cell metabolite profiling of stalk and glandular cells of intact trichomes with internal electrode capillary pressure probe electrospray ionization mass spectrometry. Anal. Chem. 88, 3049-3057, https://doi.org/10.1021/acs. analchem.5b03366 (2016).

20. Blokhina, O. et al. Ray parenchymal cells contribute to lignification of tracheids in developing xylem of Norway spruce. Plant Physiol. 181, 1552-1572, https://doi.org/10.1104/pp.19.00743 (2019).

21. Jagadish, S. V. K. et al. Physiological and proteomic approaches to address heat tolerance during anthesis in rice (Oryza sativa L.). J. Exp. Bot 61, 143-156, https://doi.org/10.1093/jxb/erp289 (2010).

22. Matsui, T., Omasa, K. \& Horie, T. High temperature-induced spikelet sterility of japonica rice at flowering in relation to air temperature, humidity and wind velocity conditions. Jpn. J. Crop Sci. 66, 449-455, https://doi.org/10.1626/jcs.66.449 (1997).

23. Pertl, H., Pockl, M., Blaschke, C. \& Obermeyer, G. Osmoregulation in Lilium pollen grains occurs via modulation of the plasma membrane H+ ATPase activity by 14-3-3 proteins. Plant Physiol. 154, 1921-1928, https://doi.org/10.1104/pp.110.165696 (2010).

24. Benkert, R., Obermeyer, G. \& Bentrup, F. W. The turgor pressure of growing lily pollen tubes. Protoplasma 198, 1-8, https://doi. org/10.1007/Bf01282125 (1997).

25. Wada, H., Matthews, M. A., Choat, B. \& Shackel, K. A. In situ turgor stability in grape mesocarp cells and its relation to cell dimensions and microcapillary tip size and geometry. Environ. Control Biol 49, 61-73, https://doi.org/10.2525/ecb.49.61 (2011).

26. Rieu, I., Twell, D. \& Firon, N. Pollen development at high temperature: From acclimation to collapse. Plant Physiol. 173, 1967-1976, https://doi.org/10.1104/pp.16.01644 (2017).

27. Wang, S. X. et al. 3D imaging of a rice pollen grain using transmission X-ray microscopy. J. Synchrotron Radiat. 22, 1091-1095, https://doi.org/10.1107/S1600577515009716(2015).

28. Yamada, N. Studies on the developmental physiology in rice pollen: I. The metabolic patterns connected with the structural changes in developing pollen. Jpn. J. Crop Sci. 41, 320-334, https://doi.org/10.1626/jcs.41.320 (1972).

29. Narayanan, S., Prasad, P. V. V. \& Welti, R. Alterations in wheat pollen lipidome during high day and night temperature stress. Plant Cell Environ. 41, 1749-1761, https://doi.org/10.1111/pce.13156 (2018).

30. Li, H. et al. Cytochrome P450 family member CYP704B2 catalyzes the $\omega$-hydroxylation of fatty acids and is required for anther cutin biosynthesis and pollen exine formation in rice. The Plant Cell 22, 173-190, https://doi.org/10.1105/tpc.109.070326 (2010).

31. Shintaku, H., Nishikii, H., Marshall, L. A., Kotera, H. \& Santiago, J. G. On-chip separation and analysis of RNA and DNA from single cells. Anal. Chem. 86, 1953-1957, https://doi.org/10.1021/ac4040218 (2014).

32. Löfke, C., Ischebeck, T., Konig, S., Freitag, S. \& Heiliviann, I. Alternative metabolic fates of phosphatidylinositol produced by phosphatidylinositol synthase isoforms in Arabidopsis thaliana. Biochem. J 413, 115-124, https://doi.org/10.1042/Bj20071371 (2008).

33. Pan, Y. Y., Wang, X., Ma, L. G. \& Sun, D. Y. Characterization of phosphatidylinositol-specific phospholipase C (PI-PLC) from Lilium daviddi pollen. Plant Cell Physiol. 46, 1657-1665, https://doi.org/10.1093/pcp/pci181 (2005).

34. Deng, X. J. et al. Phosphatidylinositol-hydrolyzing phospholipase C4 modulates rice response to salt and drought. Plant Cell Environ. 42, 536-548, https://doi.org/10.1111/pce.13437 (2019).

35. Mishkind, M., Vermeer, J. E. M., Darwish, E. \& Munnik, T. Heat stress activates phospholipase D and triggers PIP2 accumulation at the plasma membrane and nucleus. Plant J. 60, 10-21, https://doi.org/10.1111/j.1365-313X.2009.03933.x (2009).

36. Selinski, J. \& Scheibe, R. Pollen tube growth: where does the energy come from? Plant Signal. Behav. 9, e977200, https://doi.org/10. 4161/15592324.2014.977200 (2014).

37. Hakata, M. et al. Development of a new heat tolerance assay system for rice spikelet sterility. Plant Methods 13, 34, https://doi. org/10.1186/s13007-017-0185-3 (2017).

38. Wada, H. et al. Increased ring-shaped chalkiness and osmotic adjustment when growing rice grains under foehn-induced dry wind condition. Crop Sci. 51, 1703-1715, https://doi.org/10.2135/cropsci2010.08.0503 (2011). 


\section{Acknowledgements}

The authors thank Dr. Akiko Maruyama-Nakashita for helpful discussion on this work and Ms. Fujiko Komiya and Ms. Kiyomi Koga for their help in growing the rice plants and assistance with the experiments. We also thank NARO Genebank for providing seeds of N22. This work was supported by JSPS KAKENHI Grant Number $16 \mathrm{H} 02533$ and 16H04870. RE-B is a research member of National Council of Scientific and Technological Research (CONICET), Argentina.

\section{Author contributions}

H.W. conceived the study. H.W., Y.H., T.N., M.H. and K.N. performed the experiments, H.W., Y.H., and R.E.-B. analyzed data. H.W. prepared the manuscript. Y.H., T.N., H.No., R.E.-B., M.H., K.N., K.H., Y.O. and H.Na. participated in analysis of data and assisted in writing the manuscript. All authors read and approved the manuscript.

\section{Competing interests}

The authors declare no competing interests.

\section{Additional information}

Supplementary information is available for this paper at https://doi.org/10.1038/s41598-020-58869-9.

Correspondence and requests for materials should be addressed to H.W.

Reprints and permissions information is available at www.nature.com/reprints.

Publisher's note Springer Nature remains neutral with regard to jurisdictional claims in published maps and institutional affiliations.

Open Access This article is licensed under a Creative Commons Attribution 4.0 International License, which permits use, sharing, adaptation, distribution and reproduction in any medium or format, as long as you give appropriate credit to the original author(s) and the source, provide a link to the Creative Commons license, and indicate if changes were made. The images or other third party material in this article are included in the article's Creative Commons license, unless indicated otherwise in a credit line to the material. If material is not included in the article's Creative Commons license and your intended use is not permitted by statutory regulation or exceeds the permitted use, you will need to obtain permission directly from the copyright holder. To view a copy of this license, visit http://creativecommons.org/licenses/by/4.0/.

(C) The Author(s) 2020 